A PIC-Fluid Hybrid Algorithm for Multi-scale Simulations of Laser-plasma Interactions

B. I. Cohen, A. M. Dimits, F. Fiuza, A. Kemp, D. Strozzi

July 1,2013

IEEE Pulsed Power and Plasma Science San Francisco, CA, United States June 16, 2013 through June 21, 2013 
This document was prepared as an account of work sponsored by an agency of the United States government. Neither the United States government nor Lawrence Livermore National Security, LLC, nor any of their employees makes any warranty, expressed or implied, or assumes any legal liability or responsibility for the accuracy, completeness, or usefulness of any information, apparatus, product, or process disclosed, or represents that its use would not infringe privately owned rights. Reference herein to any specific commercial product, process, or service by trade name, trademark, manufacturer, or otherwise does not necessarily constitute or imply its endorsement, recommendation, or favoring by the United States government or Lawrence Livermore National Security, LLC. The views and opinions of authors expressed herein do not necessarily state or reflect those of the United States government or Lawrence Livermore National Security, LLC, and shall not be used for advertising or product endorsement purposes. 


\title{
A PIC-Fluid Hybrid Algorithm for Multi-scale Simulations of Laser-plasma Interactions
}

\author{
Bruce I. Cohen, Andris M. Dimits, Laurent Divol, Frederico R. Fiuza, Andreas J. Kemp, and David J. Strozzi
}

\begin{abstract}
A recently introduced algorithm for performing integrated kinetic simulations of the fast ignition approach to laser fusion is reviewed. The integrated algorithm uses a conventional fully electromagnetic relativistic particle-in-cell algorithm in vacuum and plasma up to a density well above cutoff for the incident laser, above which density the plasma is sufficiently collisional so that light waves and electron plasma waves are unimportant and a simplified physics model is justified that leads to improved computational efficiencies. With this tworegion algorithm integrated comprehensive kinetic simulation of fast ignition is rendered more practical. This presentation was part of a special memorial session honoring Professor C. K. (Ned) Birdsall at the IEEE Pulsed Power and Plasma Science Conference, June 17-21, 2013. Ned was a pioneer in computational plasma physics and enthusiastically championed the expansion of its use to do discovery science and to simulate plasma phenomena in a "wall-to-wall" manner.
\end{abstract}

Index Terms - plasmas, particle collisions, algorithms, computer applications, numerical analysis

$\mathrm{T}$ his conference paper was presented as part of a special memorial session honoring Professor Charles K. Birdsall. Among Ned Birdsall's many contributions to electrical engineering, plasma physics, and computational plasma physics that are especially significant are his authorship with A. Bruce Langdon of the well-known textbook Plasma Physics Via Computer Simulation (McGraw Hill, New York, 1985) [1] and Ned's more than forty years educating students at University of California Berkeley. As a graduate student at Berkeley (1970-1975), this author (B. I. Cohen) had the good fortune to take Ned's course in plasma simulation and co-

Manuscript received November 2009. This work was performed under the auspices of the U. S. Department of Energy by the Lawrence Livermore National Laboratory under Contract DE-AC52-07NA27344.

B. I. Cohen is with the Lawrence Livermore National Laboratory, P.O Box 808, Livermore, CA 94551. (phone: 925-422-9823; fax: 925-423-3484; e-mail: cohen1@1lnl.gov)

A. M. Dimits, is with the Lawrence Livermore National Laboratory, P.O. Box 808, Livermore, CA 94551. (e-mail: dimits1@1lnl.gov)

L. Divol is with the Lawrence Livermore National Laboratory, P.O. Box 808, Livermore, CA 94551. (e-mail: divol1@1lnl.gov)

F. Fiuza is with the Lawrence Livermore National Laboratory, P.O. Box 808, Livermore, CA 94551. (e-mail: fiuza1@1lnl.gov)

A. Kemp is with the Lawrence Livermore National Laboratory, P.O. Box 808, Livermore, CA 94551. (e-mail: kemp7@1lnl.gov)

D. Strozzi is with the Lawrence Livermore National Laboratory, P.O. Box 808, Livermore, CA 94551. (e-mail: strozzi2@1lnl.gov) author two of the chapters of the Birdsall and Langdon book. I was privileged to have Ned on my $\mathrm{Ph}$. D. thesis committee, and Ned had a profoundly positive influence on my professional career as a plasma physicist. Ned was one of the founding fathers of particle simulation of plasmas and vigorously participated in advancing the science of particle simulation of plasmas, establishing a mathematically rigorous foundation for it, helping to widen its scope, and educating several generations of doctoral students. One of the especially valuable skills Ned developed in his students and postdoctoral researchers was learning how to ask good scientific questions and how to evolve a question into high quality research. Ned brought an enormous energy and enthusiasm to expanding the power of particle simulation for doing discovery science. He especially loved plasma simulations that represent wall-to-wall models of an experiment, a device, or a space or astrophysical plasma. The technical content of this conference paper describes algorithms developed to facilitate integrated simulation of the plasma physics in the fast-ignition [2] approach to laser fusion and embodies Ned's philosophy on the use of particle simulation to do discovery science.

Fully electromagnetic, relativistic particle-in-cell (PIC) simulation of laser-plasma interactions has a forty-year history [1]. The simulation of the fast ignition approach to laser fusion presents extreme challenges to the traditional explicit algorithm because of the multiscale nature of the plasma the plasma density ranges from vacuum to $\sim 100 \times$ solid density in the compressed core [2]. Here we describe a PIC-fluid hybrid algorithm [3] that accommodates the multiscale nature of the physics of fast ignition and has proven successful in modeling laser-plasma interactions in fast-ignition laser fusion [4,5].

In this hybrid algorithm a traditional fully electromagnetic, relativistic, explicit PIC method $[1,6]$ is used in the vacuum region and in the plasma up to a density much greater than the critical density for the laser, e.g., $\mathrm{n}_{\mathrm{e}} / \mathrm{n}_{\mathrm{c}} \sim 100$, where $n_{e}$ is the electron density and $n_{c}$ is the critical density where the laser frequency equals the local plasma frequency. The simulations of fast ignition described in Ref. [7] and the discussion therein illustrate the enormous computational challenges presented by fast ignition, which force simulating scaled-down problems if the conventional fully electromagnetic PIC algorithm is used. In motivating the introduction of a hybrid algorithm for kinetic simulation of 
fast ignition one notes that for higher plasma densities (as long as the plasma remains sufficiently cold and collisional) an Ohm's law (electron momentum balance equation with the electron inertia dropped) is used to determine the electric field in the plasma, and inclusion of the displacement current in Ampere's law becomes optional. Dropping the displacement current eliminates light waves and electron plasma waves as justified by the high collisionality. Neglecting electron inertia to obtain Ohm's law removes the electron plasma wave regardless of whether the displacement current is retained. We note that there are still light waves and electron plasma waves at the high collisionality accompanying high density, but the waves are heavily damped (if the plasma remains relatively cold).

In the integrated hybrid algorithm [3], particles are used to represent the electrons and ions throughout the domain. In the low-density region a conventional explicit fully electromagnetic relativistic PIC algorithm is employed [1,6,7]. In the high-density hybrid region, the electrons are separated into a fast population, e.g., electrons with speeds exceeding $5 \times$ the thermal speed of the background electrons, and a colder background population. In the hybrid region, Ampere's law, (1), is used to solve for the current of the background electrons, from which the electron fluid velocity is deduced. Use of an Ohm's law, (2), with the electron inertia terms on the left side dropped, determines the electric field with moments of the particles used to evaluate terms in the Ohm's law as needed. The curl of the electric field is computed in Faraday's law, (3), from which the magnetic field is updated.

$$
\begin{gathered}
\vec{J}_{\text {cold }, e}=-\vec{J}_{\text {fast }, e}-\vec{J}_{\text {ion }}+\frac{c}{4 \pi} \vec{\nabla} \times \vec{B}-\frac{1}{4 \pi} \frac{\partial \vec{E}}{\partial t} \\
\left(\frac{m_{e}}{e}\right)\left(\frac{\partial}{\partial t}+\overrightarrow{\mathrm{v}}_{e} \cdot \vec{\nabla}\right) \overrightarrow{\mathrm{v}}_{e}=-\vec{E}+\vec{\eta}\left(\vec{J}_{\text {cold }, e}+\vec{J}_{\text {ion }}\right)-\left(e_{e}\right)^{-1} \vec{\nabla} n_{e} T_{e} \\
-\left(e n_{e} c\right)^{-1} \vec{J}_{\text {cold }, e} \times \vec{B}+\left(e n_{e} c\right)^{-1} \frac{\Delta \vec{P}_{\text {mom,coll }}^{\text {e-efast }}}{\Delta t} \\
\frac{\partial \vec{B}}{\partial t}=-c \vec{\nabla} \times \vec{E}
\end{gathered}
$$

where $\vec{J}_{s}$ is the current density for species s, $-e n_{e} \overrightarrow{\mathrm{v}}_{e}=\vec{J}_{\text {cold,e }}$, the last term on the right side of (2) is a momentum exchange term between the cold background electrons and the fast electrons due to collisions, and in the parameter regimes considered in Refs. [3, 4, 5] the resistivity is a scalar $\eta=0.51 m_{e} / n_{e} e^{2} \tau_{e i}$ and $\tau_{e i}=3 m_{e}^{1 / 2} T_{e}^{3 / 2} / 4 \sqrt{2} \ln \Lambda e^{4} Z^{2} n_{i}$. Other, more complete Ohm's laws can be considered in place of (2) as in Ref. [8]. The displacement current (last term on the right side of Eq. (1)) is typically dropped, but can be retained using a predictor-corrector iteration [3]. Omission of the electron inertia terms on the left side of (2) is justified by the dominance of the terms on the right side of the equation for the phenomena of interest in the highly collisional plasma [3]. The derivation of the two-region hybrid algorithm, an analysis of its properties, and examples of its use are given in Ref. [3].

The electric and magnetic fields are then employed to accelerate the particle momenta, and the momenta are used to advance the particle positions in the standard manner. The physics model in the high-density region employs the same physics model used earlier in studies of fast-electron transport for fast ignition in Ref. [8], except that in our model the background electrons and ions are also represented as particles. The global particle representation avoids a potential problem with an artificial source sheath that might otherwise arise at the boundary between low and high-density regions if the background plasma in the high-density region is not represented by particles. In our two-region model, the global particle representation allows for continuity of the currents across the boundary between the two regions. Our two-region algorithm relies on the fully electromagnetic, fully kinetic, first-principles model (including electron inertia) and the hybrid high-density plasma model using an Ohm's law giving the same electromagnetic fields to good approximation in the transition region. As long as the two models agree in the transition region, the integrated two-region hybrid algorithm will not be very sensitive to the precise location of the transition from the low-density model to the high-density model. Moreover, the location of the transition can be adjusted dynamically if the plasma temperature and density profiles evolve significantly.

A schematic of the hybrid algorithm is presented in Fig. 1. Implementation of the two-region hybrid algorithm in the PSC [2,3] and OSIRIS [4] electromagnetic PIC codes was relatively straightforward because the reduced Maxwell equations and Ohm's law are solved locally in space with finite-difference methods and with no global elliptic equation solve as in implicit PIC codes. Thus, the speed and excellent scaling with the number of processors of these highly optimized explicit PIC codes are retained. In the implementations and applications of the two-region hybrid algorithm $[3,4,5]$, some digital smoothing of field quantities is typically performed in the transition region between the low and high-density regions.

The two-region hybrid approach achieves improved computational efficiencies by eliminating requirements on resolving the electron Debye length, the skin depth and the plasma frequency in the high-density region where the hybrid model is used. Resolving the skin depth or Debye length in the high-density region leads to consequences for the time step in the conventional fully electromagnetic particle-in-cell algorithm because of the Courant-Friedrichs-Levy constraint: $\Delta t \leq \Delta z / c$. If a larger $\Delta z$ can be used, then a larger value of $\Delta t$ can also be used. Improved computational efficiency results because the computational costs in the simulation scale approximately as $1 /\left(\Delta t \Delta z^{r}\right)$ where $\mathrm{r}$ is the number of spatial dimensions of the simulation. In the low-density region, the usual resolution constraints must be respected [1]. The hybrid algorithm has been exploited quite successfully in simulations of fast ignition in Refs. [4,5] with a reduction in simulation costs in one-dimensional simulations exceeding an order of magnitude and two orders of magnitude or more reduction in costs in two-dimensional simulations.

Terms in the Ohm's law that derive from collisional effects, e.g., the resistivity term, must be consistent with the microscopic collision operator used for the particles. Such consistency in a PIC simulation has been demonstrated in Ref. [9]. An important aspect of the hybrid model in Refs. $[3,4]$ is 
the inclusion of an accurate and efficient Coulomb collision operator that is compatible with unequally weighted particles (used because of the large range in plasma densities $[2,3,4]$ ), which can be achieved with a variant of the Takizuka-Abe collision algorithm [10] (or the Nanbu algorithm [11]) as in Ref. [12] or in the collision algorithm extended to a grid-based model in Ref. [13]. The collision algorithm introduced in Ref. [13] involves collecting a finite number of velocity moments of the particle distribution functions locally onto the spatial grid from which an approximate velocity distribution function is constructed at the grid points. A virtual field particle is then defined for undertaking binary collisions with each test particle in a spatial cell by randomly sampling the grid-based approximate distribution function. The binary collision is then performed by using a semi-relativistic extension [13] of the Takizuka and Abe collision algorithm. The grid-based algorithm performs at least as good or better than other gridbased algorithms [14,15], is more tolerant to rare large Gaussian random number because only a scattering angle is determined by the random number, is not subject to possible noise-induced numerical instability [15], and allows use of a significantly larger time step for electron-ion collisions [13].

The algorithm reviewed here makes realistic simulation of laser-fusion fast-ignition more practical. This example of the continued growth in capability and applicability of the simulation of plasmas using particle methods would be cheered on by C. K. Birdsall.

IEEE Pulsed Power and Plasma Science Conference paper PPPS-2013 4A-5

\section{REFERENCES}

[1] C. K. Birdsall and A. B. Langdon, Plasma Physics Via Computer Simulation (McGraw Hill, New York, 1985).

[2] M. Tabak, J. Hammer, M. E. Glinsky, W. L. Kruer, S. C. Wilks, J. Woodworth, E. M. Campbell, M. D. Perry, and R. J. Mason, Phys. Plasmas 1, 1626 (1994).

[3] B. I. Cohen, A. J. Kemp, and L. Divol, J. Computational Physics 229, 4591, (2010).

[4] A. J. Kemp, B. I. Cohen, and L. Divol, Phys. Plasmas 17, 056702 (2010).

[5] F. Fiuza, M. Marti, R. A. Fonseca, L. O. Silva, J. Tonge, J. May, W. B. Mori, Plasma Physics and Controlled Fusion 53 (7), 074004 (2011).

[6] H. Ruhl, "Part I: Dynamics of Classical Many-particle Systems" in Introduction to Computational Methods in Many Body Physics, eds. Michael Bonitz and Dirk Semkat (Rinton Press, Paramus, NJ, 2006).

[7] C. Ren, M. Tzoufras, J. Tonge, W. B. Mori, F. S. Tsung, M. Fiore, R. A. Fonseca, L. O. Silva, J.-C. Adam, and A. Heron, Phys. Plasmas 13 (5), 056308 (2006).

[8] J. R. Davies, Phys. Rev. E 65, 026407 (2002); L. Gremillet, G. Bonnaud, F. Amiranoff, Phys. Plasmas 9, 941 (2002); J. J. Honrubia, C. Alfonsi'n, L. Alonso, B. Pe'rez, and J. A. Cerrada, Laser Part. Beams 24, 217 (2006); D. J. Strozzi , M. Tabak, D. J. Larson, L. Divol, A. J. Kemp, C. Bellei, M. M. Marinak and M. H. Key, Phys. Plasmas 19, 072711 (2012).

[9] B. I. Cohen, A. M. Dimits, A. Friedman, and R. E. Caflisch, IEEE Trans. Plasma Sci. 38, 2394 (2010)

[10] T. Takizuka and H. Abe, J. Comput. Phys. 25, 205 (1977).

[11] K. Nanbu, Phys. Rev. E 55, 4642 (1997).

[12] Y. Sentoku and A. Kemp, J. Comput. Phys. 227, 6846 (2008).

[13] B. I. Cohen, A. M. Dimits, and D. J. Strozzi, J. Comp. Phys. 234, 33 (2013).

[14] W. Manheimer, M. Lampe, G. Joyce, J. Comput. Phys. 138, 563 (1997)

[15] D. S. Lemons, D. Winske, W. Daughton, B. Albright, J. Comput. Physics 228 (5): 1391-1403 (2009). 
Fig. 1

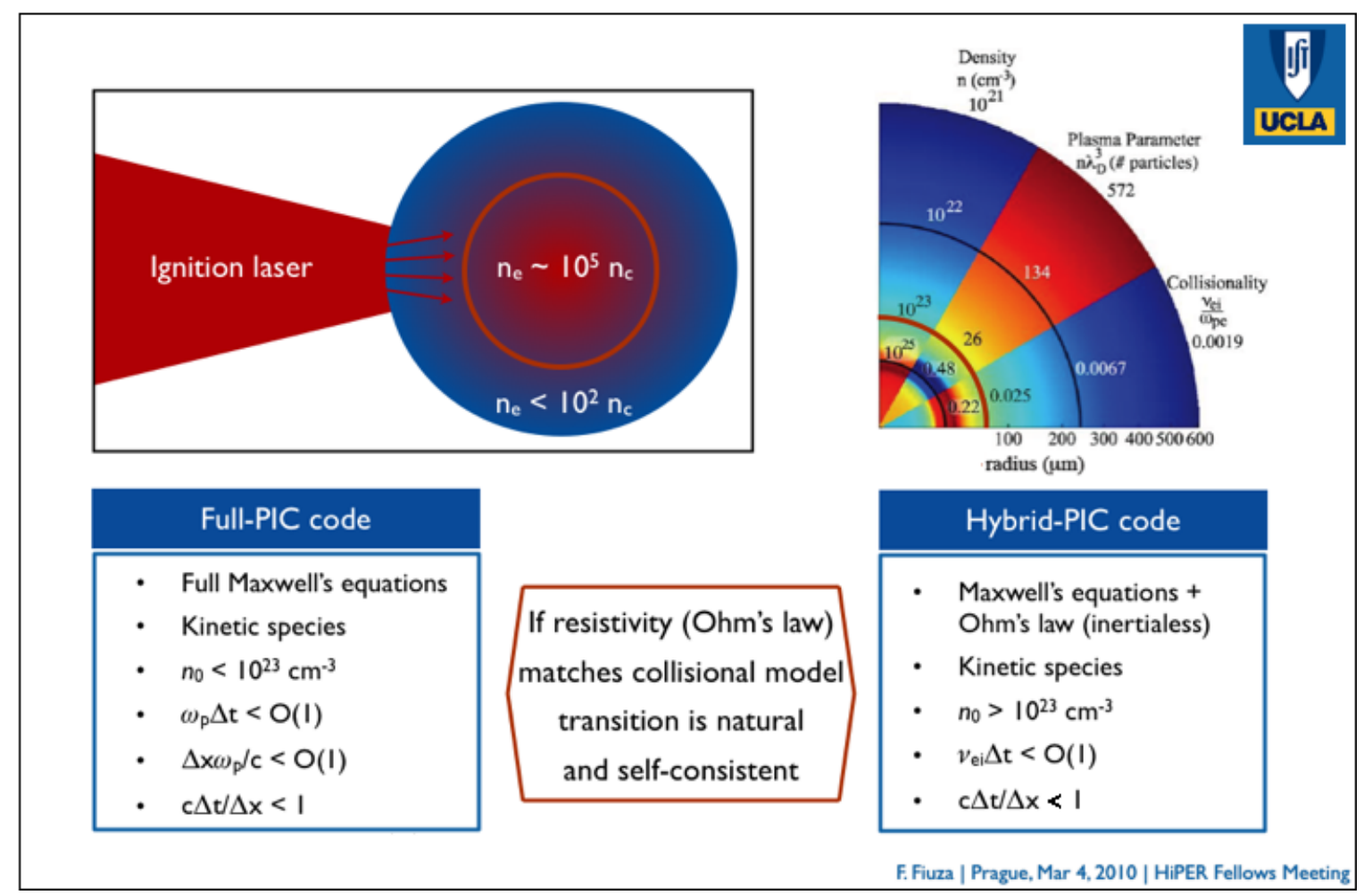

Fig. 1. Overview of two-region hybrid algorithm for simulating fast ignition. (presented in F. Fiuza, HiPER Fellows Meeting, Prague, Mar. 4, 2010 and partly adapted for use in Ref. 7) 

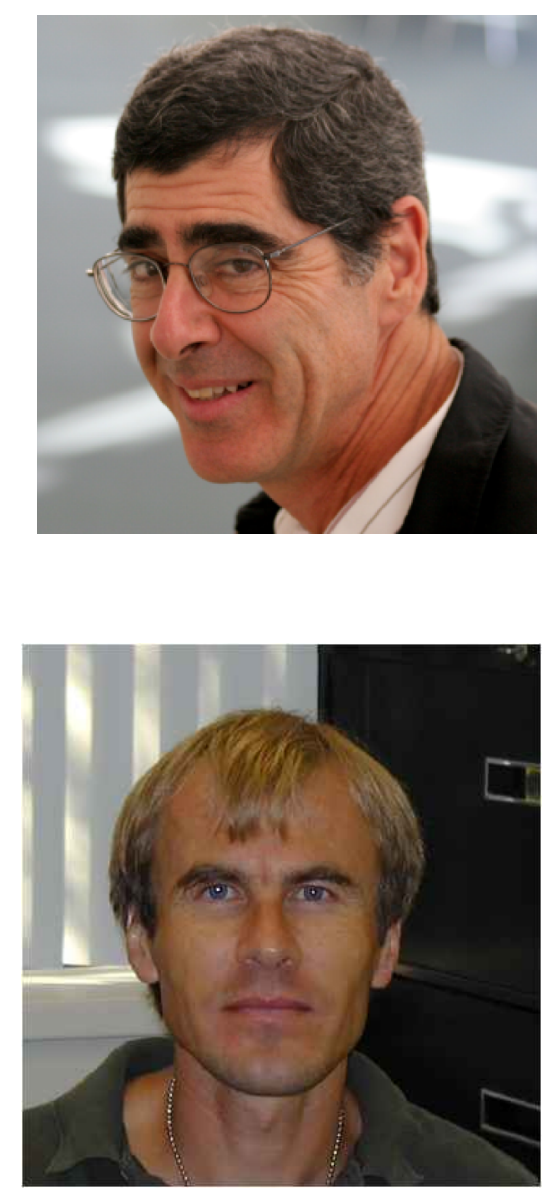

Bruce I. Cohen received his $\mathrm{Ph}$. D. degree in plasma physics from the University of California, Berkeley, in 1975. He worked at Princeton Plasma Physics Laboratory as a post-doctoral researcher from 1975 to 1976 . He is currently a research physicist and the Associate Program Leader of the Fusion Energy Theory and Computations Program at the Lawrence Livermore National Laboratory, Livermore, $\mathrm{Ca}$. His research interests are in magnetic and inertial confinement fusion, and in computational plasma physics. He is a Fellow of the American Physical Society elected through the Division of Plasma Physics.

Andris M. Dimits received his Ph. D. degree in astrophysical sciences from Princeton University in 1988. He worked at the University of Maryland as a post-doctoral researcher from 1988 to 1990 . He is currently a research physicist in the Fusion Energy Theory and Computations Program at the Lawrence Livermore National Laboratory, Livermore, Ca. His research interests are in magnetic fusion plasmas, nonlinear plasma dynamics, kinetic and fluid simulation techniques for plasmas, including derivation, implementation and application of gyrokinetic models, direct simulation and turbulence closures for fluids, and high energy density physics, including instabilities in radiative hydrodynamics. He is one of the originators of the $\delta f$ method. 